\title{
Antibodies
}

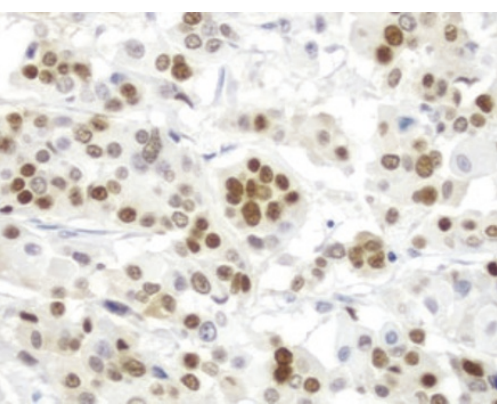

\section{Rabbit monoclonal antibodies}

Six new rabbit monoclonal antibodies have been added to Vector Laboratories' line of primary antibodies for immunohistochemical staining: CD3, COX-2, Cyclin D1, Ki67, Estrogen Receptor and Progesterone Receptor. These antibodies provide excellent results on formalinfixed, paraffin embedded tissue sections using standard immunohistochemistry methods. They can be applied to sections just like mouse monoclonals, and then detected using anti-rabbit IgG secondary detection reagents.

http://www.vectorlabs.com/

\section{Gene cloning}

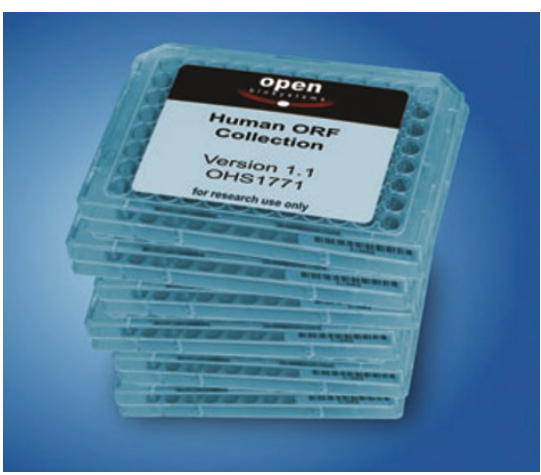

\section{Human ORF collection}

Open Biosystems offers a collection of clones containing full open reading frames (ORFs) for over 8,000 human genes, created by the Center for Cancer Systems Biology of the Dana-Farber Institute. Derived from fully sequenced Mammalian Gene Collection fulllength cDNAs, they are cloned into recombinational entry vectors. ORF clones save time by allowing users to skip PCR, cloning into an expression vector, and verifying the ends of the ORF DNA sequence. The GatewayT entry vectors ensure easy transfer into pro-

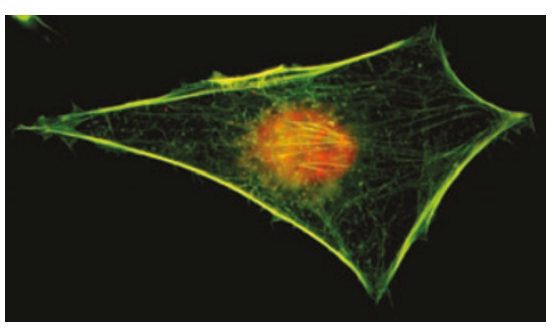

\section{Broad specificity antibodies}

Three antibodies from Upstate are validated to recognize over 80 serine/threonine and tyrosine kinases: 4G10 antibodies detect twice as many phospho-proteins as PY-20 and PT-66 using the most frequently referenced phospho-tyrosine antibody available; MPM2 antibodies are able to recognize more than 40 mitotic proteins; and highly specific phospho-MBP monoclonal antibodies and conjugates are available as detection reagents for numerous kinase assays.

http://www.upstate.com/

karyotic, mammalian, viral or insect expression systems.

http://www.openbiosystems.com/

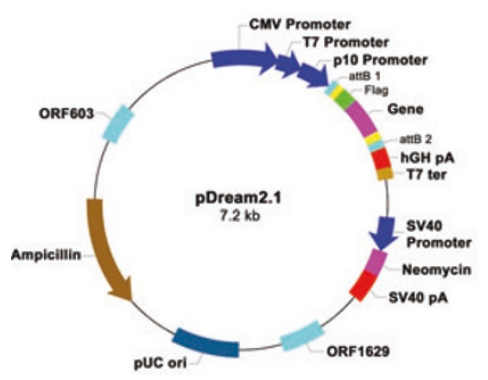

\section{Comprehensive human genes}

GenScript's GenPool ORF collection includes every single human gene with known sequence. All the ORFs are cloned into the pDream 2.1 vector, which allows expression in bacteria, Sf9 cells and mammalian cells. Each gene has a FLAG tag to facilitate purification and detection, which is also removable if needed. pDream 2.1 vector is also compatible with Gateway and LIC systems.

http://www.genscript.com/

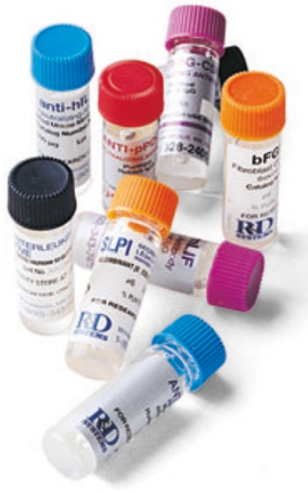

\section{Mouse anti-human antibodies}

Allophycocyanin-, fluorescein- and phycoerythrin-conjugated mouse anti-human CCL3/MIP- $1 \alpha$ antibodies are available from R\&D Systems. CCL3, a member of the CC or $\beta$-chemokine subfamily, was originally purified from the conditioned media of LPS-stimulated macrophages. It acts as a chemoattractant to a variety of cell types including monocytes, $\mathrm{T}$ cells, $\mathrm{B}$ cells and eosinophils.

http://www.RandDsystems.com/

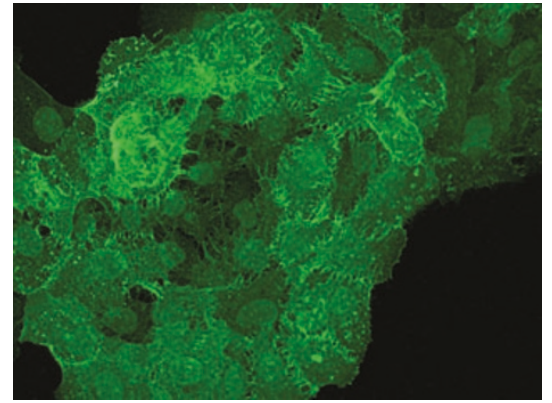

\section{Antibodies for cell-signaling research}

Epitomics' rabbit monoclonal antibodies offer more diverse epitope recognition, improved response to less immunogenic antigens, and greatly improved response to rodent proteins. All Epitomics RabMAbs offer high affinity and are extensively characterized and tested for use in WB, IHC, ICC, IF, IP and flow cytometry. Antibodies are available for key proteins involved in various cell-signaling pathways including apoptosis, cell cycle control, cytokine signaling and many phosphospecific proteins (Above: Phospho-EGFR (pY1173) RabMAb).

http://www.epitomics.com/ 